\title{
Millimeter-wave Imaging Sensor
}

\author{
Masaru Sato ${ }^{1}$ and Koji Mizuno ${ }^{2}$ \\ ${ }^{1}$ Fujitsu Ltd., ${ }^{2}$ Tohoku University \\ Japan
}

\section{Introduction}

Electromagnetic waves in the millimeter-wave band have attractive characteristics. One of their features is the wider usable frequency band compared with waves in the microwave band or lower bands. Another feature of using the millimeter-wave band is the fact that it becomes possible to design smaller and lighter equipment that utilizes that band. So it is useful to adapt millimeter waves for short-range broadband communication systems, highresolution sensing systems and radio astronomy.

In this chapter, the authors describe imaging sensors using the millimeter-wave band. In simple terms, a millimeter-wave imaging sensor is a camera that uses millimeter waves. Receivers detect the millimeter-wave energy on the imaging plane, and record the relative intensity at each pixel. Then the millimeter-wave image is reconstructed using computers as shown in Fig. 1.

The most attractive feature of millimeter waves, when compared with optical, infrared, and terahertz waves, is their ability to penetrate obstacles. And a spatial resolution higher than that achievable with microwave imaging sensors is possible. Therefore, they can be used under low-visibility conditions such as in fog, rain, dust, or fire, where optical or infrared cameras cannot be used (Mizuno et al., 2005). Their most promising application is for security. As the radiometric temperatures of an object are different depending on its metallic or dielectric properties $\left(\varepsilon_{\mathrm{r}}\right)$ and its temperature, the sensors can detect concealed weapons or explosive materials. Consequently, these sensors have undergone test installation for use in security cameras at the entrances of airports (TSA, 2009) and buildings. In addition, these sensors are also useful for finding landmines, for offering all-weather vision, for detecting cracks in exterior walls, and for screening people for skin cancer.

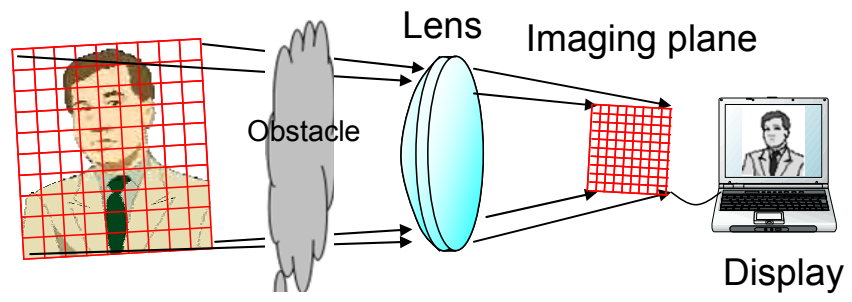

Fig. 1. Concept of millimeter-wave imaging sensor 
In section 2, the authors describe the general principle and systems for millimeter-wave imaging sensors and show the published imaging sensors that have been developed so far. Section 3 shows in detail a 94-GHz-band passive millimeter-wave imaging sensor that was developed by Fujitsu.

\section{Systems for millimeter-wave imaging sensor}

\subsection{Radiometric temperature}

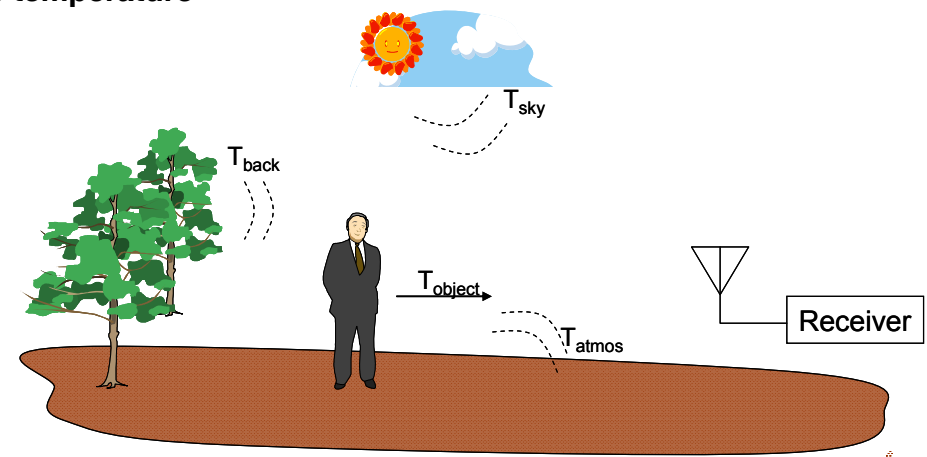

Fig. 2. Various emissions of radiometric temperatures

The total received radiometric temperature is the sum of an object's brightness, $T_{o b j}$, downwelling temperature from the sky, $T_{\text {sky, }}$, temperature from the background, $T_{\text {back, }}$, and atmospheric effects, $T_{\text {atmos }}$ as illustrated in Fig. 2.

$$
T_{\text {received }}=T_{o b j}+T_{\text {sky }}+T_{\text {back }}+T_{\text {atmos }}
$$

$-T_{o b j}$

The object's brightness temperature is comprised of the emission and reflection from the surroundings. The effective radiometric temperature is therefore:

$$
T_{o b j}=\varepsilon \cdot T_{a}+\rho \cdot T_{s u r}
$$

where $T_{a}$ is the object's temperature (in $\mathrm{K}$ ), $T_{\text {sur }}$ is the temperature of the surroundings and $\varepsilon$ is an emissivity of the object, which is a function of its dielectric properties, the roughness of its surface, and the observation angle. $\rho$ is the reflectivity of the object, and is expressed as $(1-\varepsilon)$.

A metallic object with $\varepsilon=0$ will have no emissions. But its high reflectivity will mean the second term in Equation 2 is high. For example, this will occur when $T_{\text {sur }}$ is high or the object is illuminated from millimeter-wave sources (active imaging).

On the other hand, the human body has an emissivity in the order of 0.9 .

The effective observed radiometric temperature, $T_{E}$, at a receiver is as follows:

$$
T_{E}=\frac{\varepsilon T_{a}+\rho T_{s u r}}{L_{a}} \cdot \eta
$$


where $L_{a}$ is the attenuation loss between the object and the antenna, and $\eta$ is the area ratio between the object and the main lobe of the antenna.

$-\mathrm{T}_{\text {sky }}$

$\mathrm{T}_{\text {sky }}$ is the downwelling of radiation from the sky. The sky's brightness temperature at 94 $\mathrm{GHz}$ has a value in the order of $\approx 60 \mathrm{~K}$ (Bhartia \& Bahl, 1984). In outdoor imaging, an important source of illumination is the downwelling of radiation from the sky. Typical values of the downwelling temperature as a function of the atmospheric conditions are shown in the following table.

\begin{tabular}{|c|c|}
\hline Conditions & $\begin{array}{c}\text { Downwelling } \\
\text { Temperature (K) }\end{array}$ \\
\hline Clear sky & $10-60$ \\
\hline Thick fog & 120 \\
\hline Fog & 180 \\
\hline Thick clouds & 180 \\
\hline Moderate rain & 240 \\
\hline
\end{tabular}

Table 1. Downwelling temperatures in various conditions

$-T_{\text {atmos }}$

An atmosphere acts as a blackbody and will thermally generate millimeter waves itself. This is a consequence of the reciprocity between absorption and emission. The temperature of the atmospheric emission is expressed as:

$$
T_{\text {atmos }}=T_{a}\left(1-\frac{1}{L_{a}}\right) .
$$

As is well known, atmospheric attenuation varies depending on its frequency, because electromagnetic waves are absorbed by $\mathrm{O}_{2}$ and $\mathrm{H}_{2} \mathrm{O}$, and the resonant frequencies of these are different. Figure 3 shows typical attenuations (with a water vapor density of $7.5 \mathrm{~g} / \mathrm{m}^{3}$ at $20 \mathrm{deg}$ ) at sea level (Wills, 2009).

Absorption peaks due to the vapor line exist at 22, 184, and $324 \mathrm{GHz}$. There are $\mathrm{O}_{2}$ absorption bands at 60 and $118 \mathrm{GHz}$. This large attenuation causes a large emission temperature. Here the authors assume that an object with a brightness temperature of $300 \mathrm{~K}$ and that is at a distance of $1 \mathrm{~km}$ is being observed. The attenuation loss at $60 \mathrm{GHz}$ is $13 \mathrm{~dB}$. The radiometric temperature, $T_{E}$, from the object was attenuated to $15 \mathrm{~K}$. By contrast, the emission from the atmosphere, $T_{\text {atmos, }}$ is almost $285 \mathrm{~K}$. In this case, the brightness temperature from the object is buried in the atmospheric temperature, $T_{\text {atmos. }}$.

On the other hand, there are low attenuation windows at 35, 94, 140, 220 and $360 \mathrm{GHz}$. Values for $T_{\text {atmos }}$ of these bands are relatively low and the attenuation of brightness temperature from the object is low. So these low-attenuation windows are usually used for imaging sensors. Especially, the $94-\mathrm{GHz}$-band is often used because a high spatial resolution is possible due to its short wavelength $(\approx 3 \mathrm{~mm})$. 


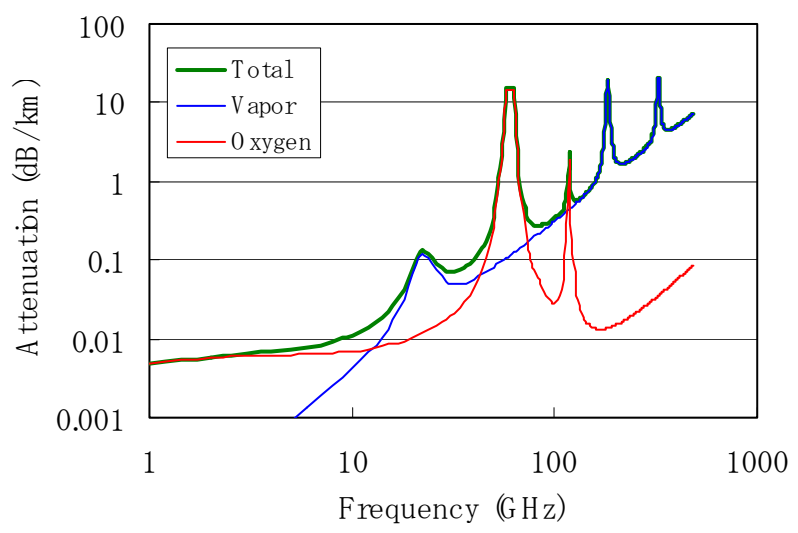

Fig. 3. Attenuations for atmospheric oxygen and water vapor

\subsection{Indoor and outdoor imaging considerations}

Fig. 4 compares the radiometric temperature of indoor and outdoor locations and shows the difference in such temperature between dielectric (a human) and metallic objects.

In outdoor imaging, $T_{s k y}$ is about $60 \mathrm{~K}$. So the effective temperatures of a human $\left(T_{\text {obj_human }}\right)$ and some metal ( $\left.T_{\text {obj_metal }}\right)$ are calculated using Equation (2) are $285 \mathrm{~K}$ and $60 \mathrm{~K}$, respectively, where the emissivity of the human and metal were set as 0.9 and 0 , respectively. As the temperature difference is $225 \mathrm{~K}$, the metallic object appears very cold in a millimeter-wave image.

By contrast, the radiometric temperature of a metallic object reflected in an indoor location, $T_{\text {obj_metal }}$, is as high as $295 \mathrm{~K}$. The temperature difference between the human and metallic object is only $13.5 \mathrm{~K}$. Thus, the millimeter-wave camera has to detect this small temperature difference in order to distinguish between the dielectric and metallic object in an indoor location.

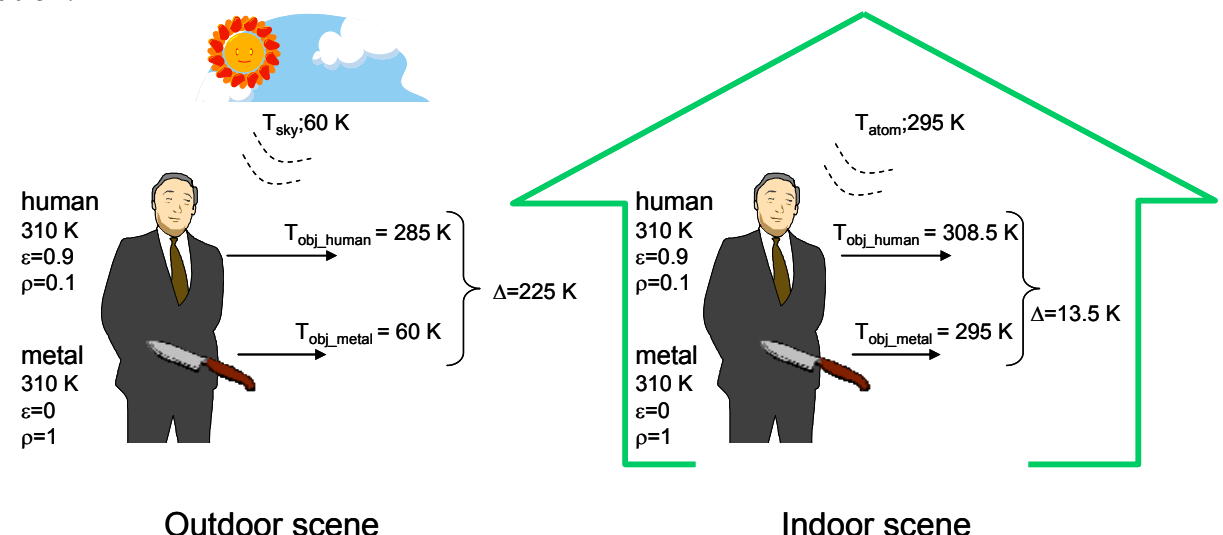

Fig. 4. Radiometric temperature observed outdoors and indoors 


\subsection{Imaging method (passive or active imaging sensor)}

Broadly speaking, imaging sensors can be classified into two types: active imaging sensors and passive millimeter-wave sensors. Fig. 5 shows simplified diagrams of these active and passive imaging sensors.
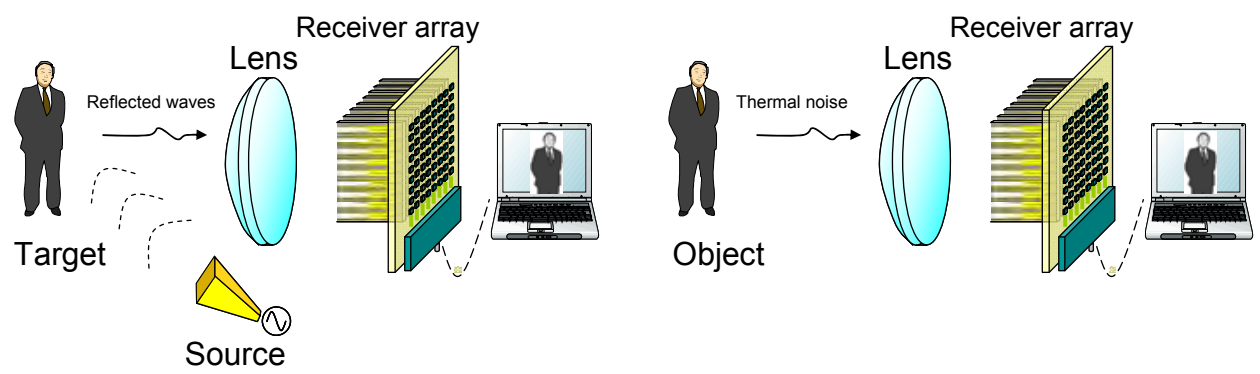

(a) Active imaging sensor

(b) Passive imaging sensor

Fig.5. Active and passive imaging sensor

An active imaging sensor radiates millimeter waves from the millimeter-wave source and illuminates the object. The receiver array observes the amplitude or phase of the reflected waves. Using these signals, the millimeter-wave image is reconstructed using a computer. Since an active imaging sensor uses a millimeter-wave source, the signal to noise ratio $(\mathrm{S} / \mathrm{N})$ received at the RX antenna is relatively high. However, the millimeter-wave image has speckle or glint when using a coherent wave as the millimeter-wave source. So, adequate signal processes are usually needed. Recently, an incoherent wave source using an impulse generator (Nakasha et al., 2007) was reported, which might be useful for solving these problems.

On the other hand, a passive imaging sensor receives incoherent millimeter waves emitted from the object. The amplitude of the radiation depends on the object's emissivity and temperature as described in section 2.1. Since passive millimeter-wave imaging sensors do not need a millimeter-wave source, the system block is simple when compared with active imaging and it lies outside the scope of the Radio Law regulations. Since the radiation from the object is the thermal noise, its power is quite small. So the receivers need to have both low noise and high sensitivity. This type of sensor is also often used in radiometers and in astronomy.

\subsection{Receiver system}

\section{-Receiver block}

Fig.6 shows various types of receiver block. Fig. 6 (a) has a traditional heterodyne structure using a mixer, which needs an LO oscillator. The recent development of a low-noiseamplifier (LNA) using InP, GaAs HEMTs or SiGe MMIC and a square-law detector have made it possible to use the direct detection as shown in Fig. 6 (b). An LNA boosts the signal above the detector's noise floor. Furthermore, Sb-based heterostructure diodes (Siegwart et al., 2006) with high sensitivity have a possibility to remove the need for pre-amplification, which may lead to the development of imaging systems that are compact and inexpensive. 


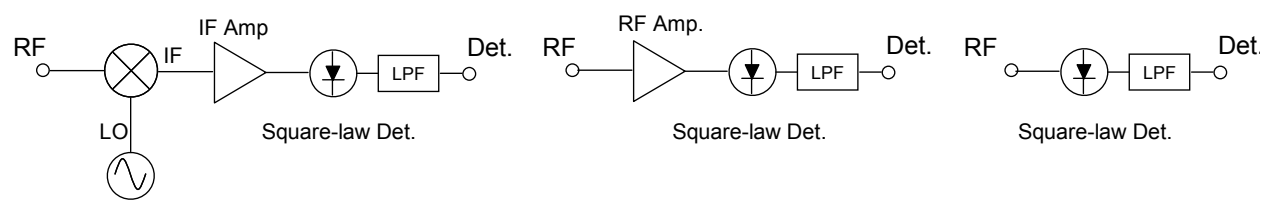

(a) Heterodyne detection

(b) Direct detection with peramplifier

(c) Direct detection

Fig. 6. Receiver block diagram

RF Amp.

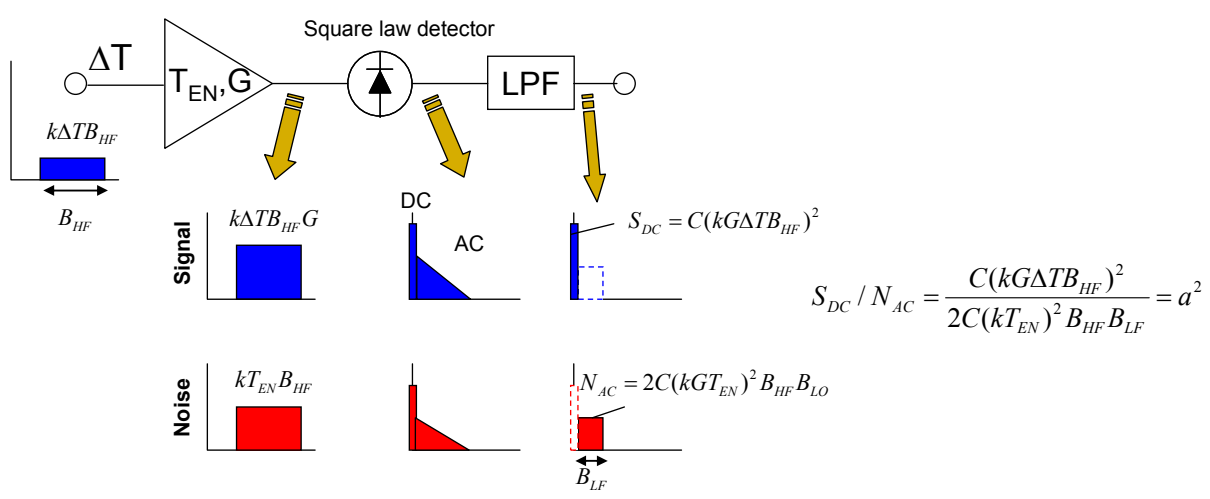

Fig. 7. Receiver block diagram and power spectrum at each stage

\section{-Total power receiver}

Here, the authors consider the relationship among minimum detectable temperature, noise figure, and integration time, $\tau$, of a receiver using the direct detection with a preamplifier, as shown in Fig. 6 (b).

Fig. 7 shows a receiver block diagram. The brightness temperature of an object, $\Delta \mathrm{T}$, is observed and amplified in an LNA, then fed into a square-law detector. In the LNA, noise is also produced. The equivalent noise temperature, $T_{E N}$, is expressed using a noise factor, $F$ and ambient temperature, $T_{a}$.

$$
T_{E N}=(F-1) \cdot T_{a} .
$$

At the square-law detector, signals from the object and the noise produced in the LNA are converted to both DC and AC components. The DC component will be displayed as millimeter-wave intensity of the object. DC component of the detected signal is expressed by (Tiuri, 1964)

$$
S_{D C}=C\left(k G \Delta T B_{H F}\right)^{2}
$$

where $k$ is the Boltzmann constant, $B_{H F}$ is the bandwidth of the LNA and detector, and C is a constant that relates to the sensitivity of the detector. Although both DC and AC components are produced from the noise, the DC components of the noise can be removed by applying a proper offset voltage. An AC component of the signal also exists, its amount is small compared with the AC component of the noise. 
The AC component converted from the noise at the output of LPF, which causes fluctuation in the detected voltage, is given by

$$
N_{A C}=2 C\left(k G T_{E N}\right)^{2} B_{H F} \cdot B_{L F}
$$

where $B_{L F}$ is the bandwidth of the LPF.

So, the ratio of the DC component of the signal to the AC component of the noise determines the signal to noise ratio $(\mathrm{S} / \mathrm{N})$. Here, the authors define the $\mathrm{S} / \mathrm{N}$ as $a^{2}$.

$$
S_{D C} / N_{A C}=\frac{C\left(k G \Delta T B_{H F}\right)^{2}}{2 C\left(k G T_{E N}\right)^{2} B_{H F} B_{L F}}=a^{2} .
$$

Using Equations (5) and (8), the relation between the bright temperature difference and the noise factor of the receiver is expressed in Equation (9):

$$
F=1+\frac{\Delta T}{a T_{a}} \cdot \sqrt{\frac{B_{H F}}{2 B_{L F}}} .
$$

The relation between the integration time, $\tau$, and the bandwidth of the LPF based on an R-C circuit is given by:

$$
B_{L F}=\frac{1}{2 \pi \tau}
$$

Using Equation (9), the noise factor will therefore be:

$$
\begin{gathered}
F=1+\frac{\Delta T}{a T_{a}} \cdot \sqrt{\pi \cdot \tau \cdot B_{H F}} \\
\Delta T=\frac{a T_{a}(F-1)}{\sqrt{\pi \cdot \tau \cdot B_{H F}}} .
\end{gathered}
$$

Equation (12) indicates that a larger $B_{H F}$ with a smaller $F$ of the receiver produces a smaller detectable temperature, $\Delta \mathrm{T}$, which means an improved temperature resolution in the millimeter-wave image. Although a longer integration time will also make it possible to have a smaller $\Delta \mathrm{T}$, it takes longer until the detected voltage is settled, which means that it would take a long time to acquire an image.

\section{-Dicke receiver}

A Dicke receiver (Tiuri, 1964) is well-known architecture for decreasing fluctuations in a detected voltage. These fluctuations are caused from gain or temperature variations in the LNA or DC drift in the detector (1/f noise). A Dicke receiver consists of an RF switch, an LNA, a detector and a lock-in amplifier, as shown in Fig. 8. The RF switch alternately switches between the millimeter-wave signal from the scene and the reference noise at a repetition frequency of $f_{M}$. The output switch located in the lock-in-amplifier switches in synchronism with the antenna switch. By subtracting the received voltage in a lock-in 
amplifier, fluctuations in the detected voltage can be decreased, if $f_{m}$ is high enough in comparison with the gain instability frequencies.

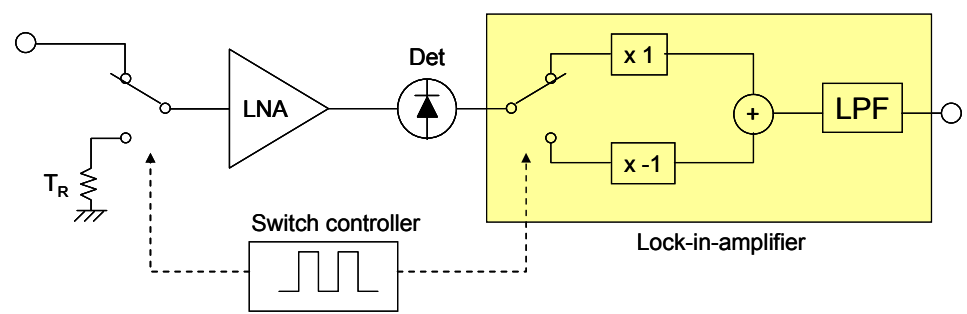

Fig. 8. Block diagram of a Dicke receiver

In the Dicke receiver, a receiver observes signals only half of the time, assuming there is a square wave modulation. The bandwidth of the receiver, $B_{H F}$ is halved. Equation (6) is modified as:

$$
S_{D C}^{\prime}=C\left(\frac{k G \Delta T B_{H F}}{2}\right)^{2} .
$$

On the other hand, the AC component converted from the noise is the same as in Equation (7) due to the halved integration time in LPF. So the noise factor of a Dicke receiver is modified as follows:

$$
F^{\prime}=1+\frac{\Delta T}{2 a T_{a}} \cdot \sqrt{\pi \cdot \tau \cdot B_{H F}}
$$

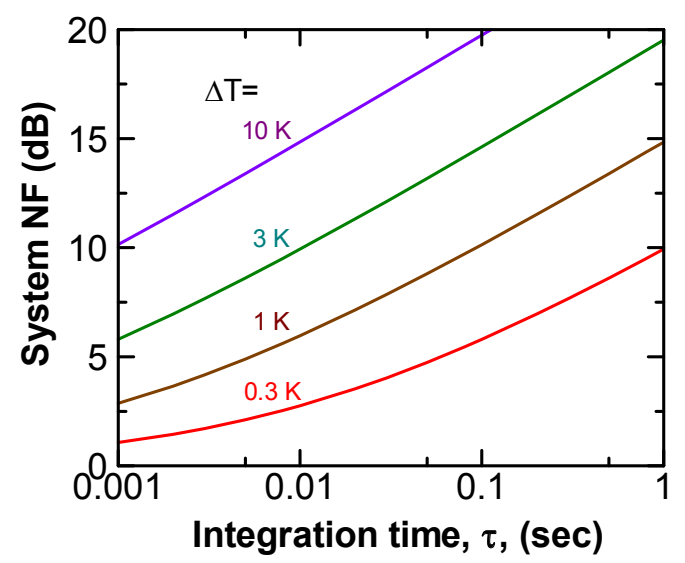

Fig. 9. System NF dependence on integration time

Fig. 9 shows the dependence of the system noise figure on integration time with various minimum detectable temperatures, $\Delta \mathrm{T}$, where an ambient temperature of $300 \mathrm{~K}$, a bandwidth of $10 \mathrm{GHz}$, and an $a$ of 10 have been set. When observing millimeter waves in 0.01 second with $\Delta \mathrm{T}$ of $1 \mathrm{~K}$, the required system noise should be within $6 \mathrm{~dB}$. 


\subsection{Scanning method}

The image acquisition time is composed of the integration time in the receiver and the time for moving the receivers. An X-Z mechanical scanning method is shown in Fig. 10 (a). It takes a long time to acquire an image with this method because the movement in the focal plane is large. The scanning method shown in Fig. 10 (b) uses a mirror. By moving the mirror slightly, the scanning direction can be changed. Fig. 10 (c) shows a frequency scanned antenna that uses a leaky-wave guide (Kuki, 2008). The angle of radiation depends on the frequency. Therefore, by scanning the amplitude on each frequency, a onedimensional scan is possible using this antenna.

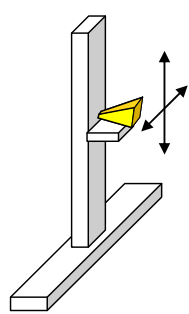

(a) X-Z mechanical scan

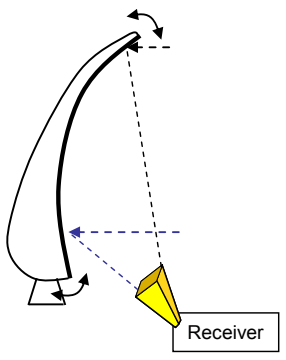

(b) Mechanical scan (Mirror)

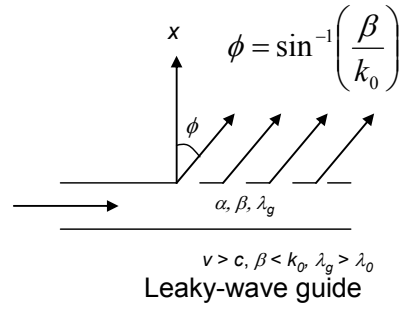

(c) Frequency scanned antenna

Fig. 10. Scanning method for millimeter-wave imaging

2.6 Published performances of millimeter-wave imaging sensors

Table 2 shows the published performances of millimeter-wave images.

\begin{tabular}{|c|c|c|c|c|c|c|c|}
\hline & \begin{tabular}{|l|} 
Active/ \\
Passive \\
\end{tabular} & $\begin{array}{r}\text { Freq. } \\
(\mathrm{GHz})\end{array}$ & $\begin{array}{c}\text { Scan } \\
\text { method }\end{array}$ & Sensitivity & $\begin{array}{l}\begin{array}{l}\text { Pixel } \\
\text { format }\end{array} \\
\end{array}$ & $\begin{array}{r}\begin{array}{r}\text { Frame rate } \\
\text { (frame } / \mathrm{s} \text { ) }\end{array} \\
\end{array}$ & Ref \\
\hline Trex & Passive & 75-93 & $\begin{array}{r}\text { Frequency + } \\
\text { mechanical }\end{array}$ & $2-3 \mathrm{~K}$ & $128 \times 60$ & 0.5 & $\begin{array}{l}\text { (Kolinko et } \\
\text { al., 2005) }\end{array}$ \\
\hline Millivision & Passive & 100 & Mechanical & - & - & 10 & $\begin{array}{c}\text { (Millivision, } \\
\text { 2009) }\end{array}$ \\
\hline QinetiQ & Passive & 35 & Mechanical & $0.28 \mathrm{~K}$ & $32 \times 32$ & 1 & $\begin{array}{c}\text { (Sinclair et } \\
\text { al., 2001) }\end{array}$ \\
\hline $\begin{array}{l}\text { Northrop } \\
\text { Grumman }\end{array}$ & Passive & $84-94$ & Mirror & $2 \mathrm{~K}$ & $30 \times 20$ & 17 & $\begin{array}{c}\text { (Yujiri, } \\
2006)\end{array}$ \\
\hline $\begin{array}{c}\text { Farran } \\
\text { Technolog } \\
y\end{array}$ & Passive & 94,140 & Mirror & $1 \mathrm{~K}$ & $83 \times 50$ & 10 & $\begin{array}{c}\text { (Vizard \& } \\
\text { Doyle, } \\
2006 \text { ) }\end{array}$ \\
\hline NHK & Active & $60-62$ & $\begin{array}{l}\text { Frequency+ } \\
\text { Mechanical }\end{array}$ & - & $30 \times 20$ & 0.1 & (Kuki, 2008) \\
\hline $\begin{array}{c}\text { Tohoku } \\
\text { University }\end{array}$ & Passive & $30-40$ & Imaging Array & $1 \mathrm{~K}$ & $6 \times 6$ & 10 & $\begin{array}{l}\text { (Mizuno et } \\
\text { al., 2009) }\end{array}$ \\
\hline Fujitsu & Passive & 84-99 & Mechanical & $1 \mathrm{~K}$ & $40 \times 40$ & 0.1 & $\begin{array}{c}\text { (Sato et al., } \\
\text { 2009) }\end{array}$ \\
\hline
\end{tabular}

Table 2. Published performances of millimeter-wave imaging sensors 


\section{94-GHz-band PMMW imaging sensor}

This section describes a 94-GHz-band passive millimeter-wave imaging sensor that was developed by Fujitsu. Fig. 11 shows a block diagram of the imaging system. In section 3.1 the authors describe the design and performance of the receiver MMIC based on InP HEMT technology. The development of the antipodal linearly tapered slot antenna (LTSA) and lens that are well suited for millimeter-wave sensors is shown in sections 3.2. and 3.3, respectively. Finally, the authors show an example of a millimeter-wave image captured by their imaging system.

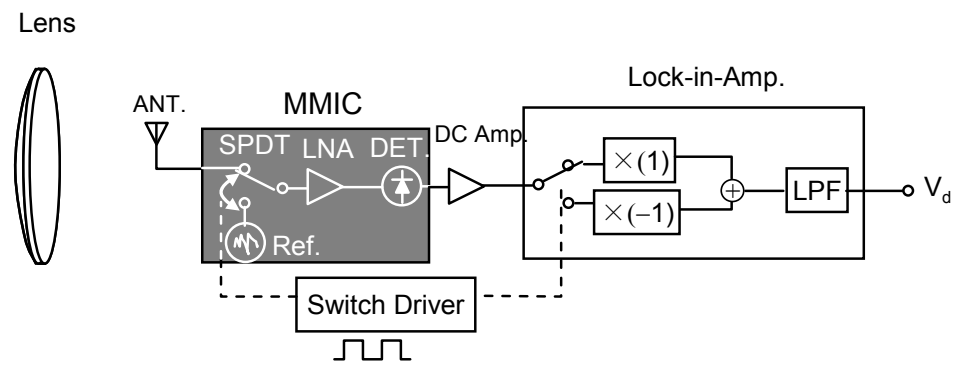

Fig. 11. Block diagram of passive millimeter-wave imaging sensor developed by Fujitsu.

\subsection{Receiver MMIC}

The most challenging component to develop is the receiver MMIC. Especially an LNA is one of the key components. In order to detect a millimeter-wave signal in a short integration time without deteriorating the system noise figure, the NF of LNA should be extremely low. In addition, a gain of over $30 \mathrm{~dB}$ is required to provide a sufficient boost for the detector. The authors designed and fabricated an ultra-low-noise, high-gain LNA using InP HEMT. The authors also developed an SPDT switch and detector and integrated them into a single chip.

\section{-InP HEMT technology}

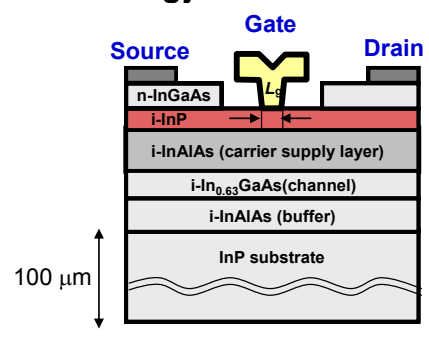

(a) Schematic of the InP HEMT technology

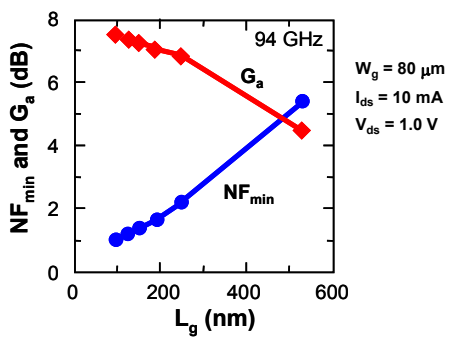

(b) Noise performance on gate length

Fig. 12. Device structure and noise performance of InP HEMT technology

To meet these requirements, the authors used InP HEMT technology. The epitaxial wafer consists of an InGaAs channel layer and an InAlAs carrier supply layer. The gate had a Tshaped $\mathrm{Ti} / \mathrm{Pt} / \mathrm{Au}$ structure which was fabricated using electron beam (EB) lithography. The 
thickness of the InP substrate was thinned to $100 \mu \mathrm{m}$. A schematic of the InP HEMT is shown in Fig. 12 (a).

Fig. 12 (b) shows the dependence of the $N_{\text {min }}$ and the associated gain, $G_{a}$, on the gate length at a frequency of $94 \mathrm{GHz}$. The gate width was $2 \times 40 \mu \mathrm{m}$, and the applied bias current, $\mathrm{I}_{\mathrm{ds}}$, and voltage, $\mathrm{V}_{\mathrm{ds}}$, are $10 \mathrm{~mA}$ and $1.0 \mathrm{~V}$, respectively. Both the noise figure and associated gain have significant dependence on gate length. By decreasing the gate length to $100 \mathrm{~nm}$, the authors could obtain an $\mathrm{NF}_{\min }$ of $1 \mathrm{~dB}$ and an associated gain of $7.5 \mathrm{~dB}$.

Schottky diodes constructed using HEMT technology were used for the detector. The gate length and width were $4 \mu \mathrm{m}$ and $5 \mu \mathrm{m}$, respectively.

\section{-LNA}

The LNA requires a gain of over $30 \mathrm{~dB}$, but it is difficult for a single amplifier to obtain such a high gain while maintaining stable operation. In the worst case, the amplifier oscillates. This instability is caused not only by feedback in the circuitry but also by the feedback path transmitted from the output to the input terminals via the MMIC (InP) substrate (Sato et al., 2007).

The authors estimated the feedback power that was transmitted in the MMIC substrate using a 3D electromagnetic simulation (HFSS). First, the authors extracted structure models from CAD data of the MMIC, from which the active devices were removed. Next, the authors simulated the feedback power from the output to the input terminals. Fig. 13 shows an E-field simulation of the feedback power at a frequency of $94 \mathrm{GHz}$. In this simulation, a conventional thin film microstrip structure for the transmission line was used, as shown in Fig. 14 (a). The blue shade in Fig. 13. represents the feedback power. Because a semiconductor's substrate has high resistivity, electromagnetic waves can transmit in the substrate at high frequency. As the MMIC is mounted on a metal plate, most of the InP substrate was sandwiched between the second metal and the metal substrate. So the parallel-plate mode was excited, which caused the feedback. Dasded line in Fih. 14. (d) shows the feedback power. About $-20 \mathrm{~dB}$ feedback power was obserbed at W-band. And that feedback caused instability in the high-gain amplifier.

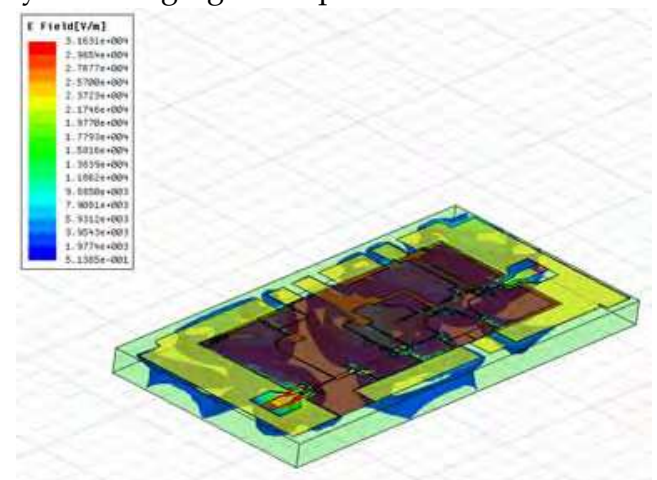

Fig. 13. E-field simulation of the feedback power at a frequency of $94 \mathrm{GHz}$

To isolate this feedback path, Tessmann et al. in 1997 reported the structure to stabilize it by thinning the MMIC's substrate to $50 \mu \mathrm{m}$ and mounting them on an absorbing material. But with this method it is difficult to assemble the MMIC. 
The authors proposed two structures. One is illustrated in Fig. 14 (b). An MMIC is flipchipped on the interposer. In the MMIC, the authors employed an inverted microstrip line (IMSL). As the back side of the MMIC is exposed to the air, the parallel plate mode is hard to excite. The feedback power was decreased drastically as shown in Fig. 14 (d). In addition, the authors embedded a resister layer in the areas except for the transistors and transmission line as shown in Fig. 14 (c). Using this resistor layer, an enhancement of about $5 \mathrm{~dB}$ is realized. Using this structure, it is possible to realize an amplifier with a gain of over $30 \mathrm{~dB}$.

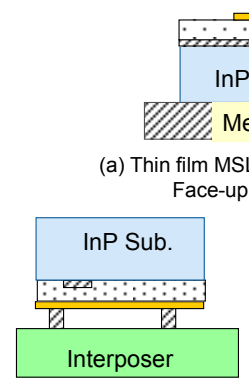

(b) IMSL based MMIC for FCB assembly (c) IMSL with resistor layer MMIC for FCB assembly

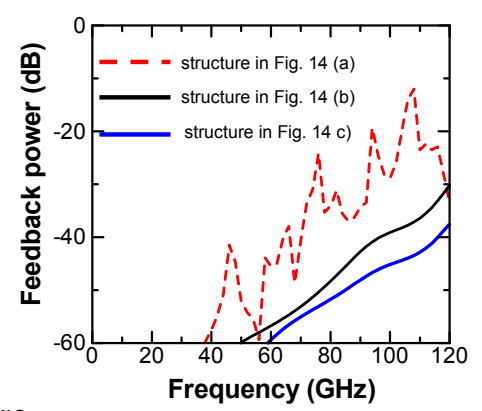

(d) Simulated feedback power of MMIC

Fig. 14. Cross-sectional view of MMICs and simulated feedback power

Using these techniques, the authors designed and fabricated the LNA. The circuit consists of a 7-stage common-source amplifier. The gate widths of the transistors in each stage were $2 \times$ $20 \mu \mathrm{m}$, the total power consumption was $60 \mathrm{~mW}$, and the overall chip size was $2.5 \times 1.2 \mathrm{~mm}^{2}$. Fig. 15(a) shows the measured S-parameters of the LNA. A linear gain of $35 \mathrm{~dB}$ was achieved between 90 and $110 \mathrm{GHz}$, and the measured input and output return loss in this frequency range was below $8 \mathrm{~dB}$. In addition, the measured $S_{12}$ was below $-40 \mathrm{~dB}$. Thus, the MMIC structure using IMSL with a resistor layer can be used in high-gain amplifiers. The authors also measured the noise figure of the LNA as shown in Fig. 15 (b), which was about $4 \mathrm{~dB}$. This high gain with low-noise performance achieved in the 130-nm InP HEMTs indicates the LNA can be used for a single-chip MMIC.

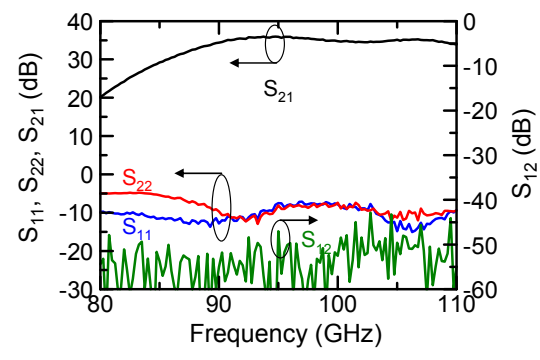

(a) Measured S-parameters

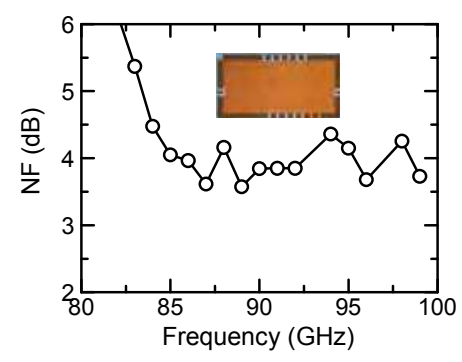

(a) Measured noise figure

Fig. 15. Measured S-parameters and noise figure of the LNA 


\section{-Detector}

A Schottky diode constructed on a HEMT device was employed for the detector. Although Schottky diodes are usually biased to increase the sensitivity, this causes an increase in noise. The authors chose a diode's dimension of $\mathrm{Lg}=4 \mu \mathrm{m}$ and $\mathrm{Wg}=5 \mu \mathrm{m}$ so that it could be used in a zero-bias condition. The sensitivity of the diode itself was $150 \mathrm{~V} / \mathrm{W}$. In order to increase the sensitivity, LNA was integrated with the detector, which will be discussed below.

\section{-SPDT switch}

An SPDT switch was used to control the direction between the antenna and reference noise. Fig. 16 (a) shows a schematic diagram of the distributed single-pole-double-throw (SPDT) switch, which is composed of two single-pole-single-throws (SPST) switches and two quarter-wavelength impedance transformers. The drain terminals of the transistors were connected to the transmission lines periodically. One port of the SPST switch was terminated in $50 \Omega$ as a reference load. In addition, the authors added an RF choke and a current source to adjust the noise power from the reference load to be equal to the antenna noise.

The gates of the transistors in each SPST switch were biased below the pinch-off voltage; for example $-1 \mathrm{~V}$, or alternately biased in the linear region such as at $0.5 \mathrm{~V}$. When the bias applied is below the pinch-off voltage, the transistors act as capacitors. The authors chose the length and width of the transmission lines to give characteristic impedance $Z_{0}$ for the artificial transmission line of $50 \Omega(\mathrm{ON}$-state). On the other hand, when the bias applied is a positive voltage such as $0.5 \mathrm{~V}$, the transistors act as small resistances. Since the transistors are shunted between the transmission lines, the SPST circuit works as a short circuit (OFFstate).

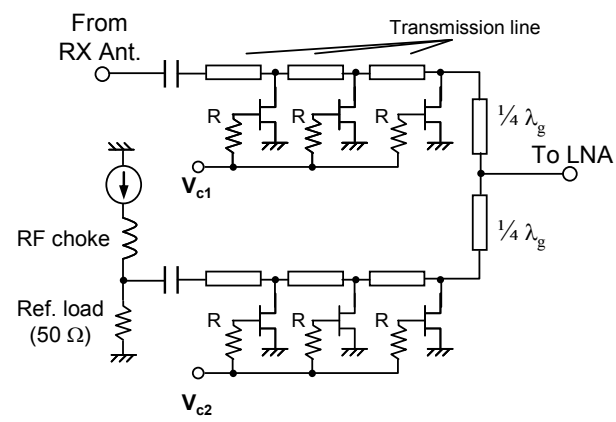

(a) Schematic diagram

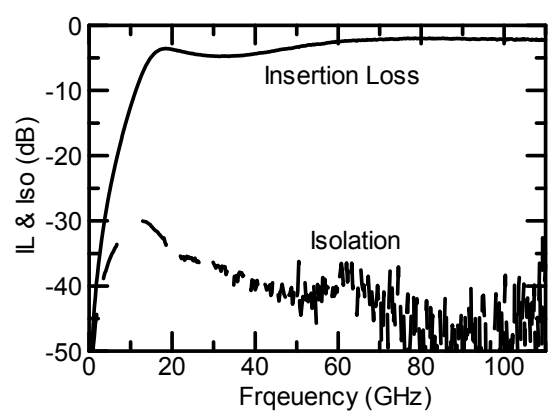

(b) Measured insertion loss and isolation

Fig. 16. Schematic diagram and measured performance of SPDT switch

Using this technique, the authors fabricated an SPDT switch using InP HEMT technology. Fig. 16 (b) shows the insertion loss and isolation of the developed SPDT switch. The gate bias condition was $-1 \mathrm{~V}$ for the ON-branch, and $0.5 \mathrm{~V}$ for the OFF-branch. The measured insertion loss was within $2 \mathrm{~dB}$ between 60 and $110 \mathrm{GHz}$ and the isolation was better than $-40 \mathrm{~dB}$. 


\section{-Single chip receiver MMIC}

The authors integrated the switch, LNA, and detector onto a single MMIC. The die photo and measured performance of the receiver MMIC is shown in Fig. 17. The chip size is $2.5 \times$ $1.2 \mathrm{~mm}^{2}$. The total power consumption was $64 \mathrm{~mW}$.

Here are the measured results of the MMIC. The authors measured the sensitivity of the detector MMIC by calculating the ratio of the detected voltage, $\mathrm{V}_{\text {det }}$, to the input millimeterwave power, $P_{\mathrm{in}}$. In this measurement, the authors used a $94-\mathrm{GHz} \mathrm{CW}$ source as the input. The bias voltages for the SPDT switch, $\mathrm{V}_{\mathrm{c} 1}$ and $\mathrm{V}_{\mathrm{c} 2}$ were -1 and $0.5 \mathrm{~V}$, respectively while the MMIC receiver was measuring the millimeter-wave signal (measuring mode). When the receiver was measuring the reference load (reference mode), $V_{c 1}$ and $V_{c 2}$ were set to 0.5 and $-1 \mathrm{~V}$, respectively. As the isolation of the SPDT switch was better than $-40 \mathrm{~dB}$, the difference in the detected voltage between the measuring and reference modes was detectable under $-70 \mathrm{dBm}$. Because the amplitude of the millimeter waves $\left(k T B_{H F}\right)$ was around $-70 \mathrm{dBm}$ when the bandwidth was set to $10 \mathrm{GHz}$, it is possible to detect millimeter waves using the receiver MMIC.

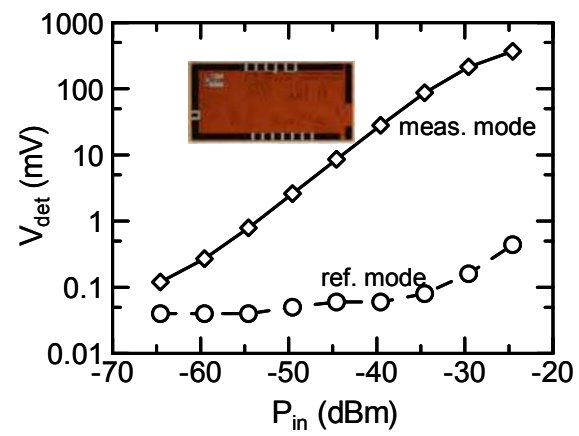

Fig. 17. Measured detedted voltage as a function of input millimeter-wave power

\subsection{Antipodal linearly tapered slot antenna}

To collect 2D mapping of millimeter-wave energy, the authors placed receivers at the focus of the imager's lens and mechanically scanned the receivers on the focal plane. The resulting system can produce high-spatial-resolution images because the lens-coupled antenna has high directivity and a low sidelobe level. By using an array of receivers, the image collection time can be reduced by a factor equal to the number of receivers. To increase the number of receivers, the size of each receiver must be miniaturized. Although a waveguide horn antenna is usually used in front of the receivers, it is too large to be used in this case which makes it difficult to achieve a high-density receiver array.

The authors have developed a small tapered-slot antenna (Sato et al. 2008) called an antipodal linearly tapered-slot antenna (LTSA) with an aperture size as small as $1.2 \lambda_{0}$ square, where $\lambda_{0}$ is the wavelength in a vacuum at the centre frequency. The antenna has almost the same E-plane and H-plane patterns (circular radiation pattern), which make it well suited for use in a lens-coupled antenna. In addition, the antenna has a microstrip interface. Then the antenna and MMIC are connected by a low-cost flip-chip bonding assembly. The authors will discuss how to design this system to have an antenna that can obtain circular radiation patterns and have a low sidelobe level. 
Fig. 18 shows the geometry of the antenna with a compact microstrip-slot transition. A metal pattern that forms the tapered-slot section is printed on the top and bottom sides of the dielectric layer. The slot profile is defined by the linear function

$$
f(x)=\frac{W \cdot x}{2 L}
$$

where $\mathrm{L}$ and $\mathrm{W}$ are the antenna length and aperture width, respectively. Corrugated patterns are used on both sides of the metal edge. Corrugations work to suppress the surface-mode waves excited on the dielectric substrate and eventually widen the effective aperture size of the antenna. The dimensions of a corrugation with a width $\varpi_{c}$, pitch $p$ and length $l_{c}$ were reported by Sato et al. in 2004 to be

$$
\omega_{\mathrm{c}}=0.034 \lambda_{0}, \mathrm{p}=0.069 \lambda_{0}, 1_{\mathrm{c}} \cdot 0.116 \lambda_{0}
$$

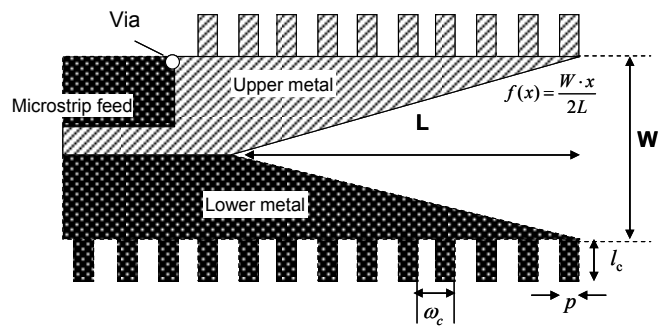

Fig. 18. Geometry of the linearly tapered slot antenna

The radiation pattern of the LTSA can be controlled by changing L, W, the substrate thickness and the dielectric constant. To optimize these parameters in a shorter time, an antenna with fewer parameters must be developed.

The authors now turn to the design procedure to obtain a circular radiation pattern using $3 \mathrm{D}$ electromagnetic simulation (HFSS). In their simulation, the authors used a $125-\mu \mathrm{m}$ thick dielectric substrate $\left(\varepsilon_{\mathrm{r}}=2.9\right)$. The antenna radiation patterns were controlled by two parameters: antenna length L and width of aperture. Fig. 19 (a) shows the simulated 10-dBbeam width of the radiation pattern as a function of $\mathrm{L}$, when the width of the aperture, $\mathrm{W}$, is $0.78 \lambda_{0}$. As the antenna length increased, the beam width in both the E- and H- planes decreased drastically. The authors chose an antenna length of $4 \lambda_{0}$ because it gives almost the same width in the E- and H-planes.

Fig. 19 (b) shows the simulated results for the 10-dB-beam width against the width of the aperture $\left(0.3-1.3 \lambda_{0}\right)$ when the antenna length, $L$, is $4 \lambda_{0}$. As the width of the aperture increased, the E-plane beam width decreased, but the H-plane beam width remained the same. Almost identical beam widths of $50.8 \mathrm{deg}$ can be obtained by choosing a $\mathrm{W} / \lambda_{0}$ of 0.76 . It can be seen in Figs. 19 (a) and (b) that the antenna length, L, rough-tunes the beam width, and the width of the aperture, $\mathrm{W}$, fine-tunes it. Using this design procedure, the authors were able to design a circular radiation pattern that is well suited to their lens-coupled PMMW imaging system. 


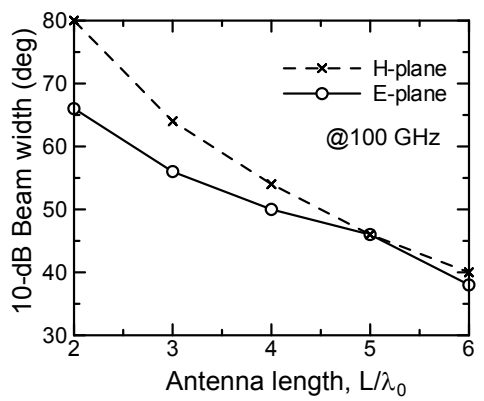

(a) 10-dB beam width as a function of $\mathrm{L}$

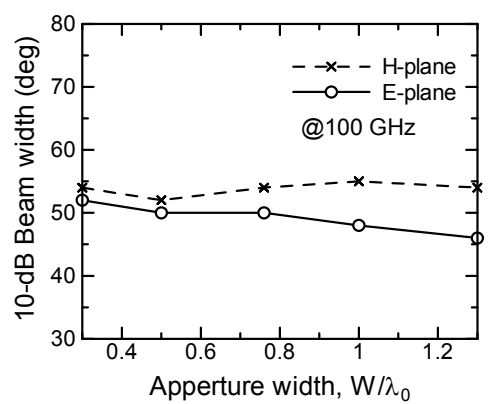

(b) 10-dB beam width as a function of $\mathrm{W}$

Fig. 19. Simulated 10-dB beam width of radiation patterns as a function of antenna length (a) and aperture width (b)

The authors fabricated an antipodal LTSA with a compact microstrip-slot transition using a PTFE substrate (Duroid 6002) with a thickness of $125 \mu \mathrm{m}$. The authors chose $\mathrm{L}$ and $\mathrm{W}$ of $4 \lambda_{0}$ and $0.78 \lambda_{0}$, respectively, to obtain the circular radiation pattern. The size of the antenna was $16 \times 3.7 \mathrm{~mm}$. Fig. 20 (a) shows a photograph of the antipodal LTSA. The authors mounted the antenna on a module with a W-1 connector. The measured and simulated radiation patterns are shown in Fig. 20 (b). The operating frequency was $94 \mathrm{GHz}$ for both. The authors were able to obtain circular radiation patterns using the proposed design procedure. The measured 10-dB-beam widths of the E- and H-plane patterns were 50 and 54 deg respectively. In addition, the sidelobe level of the E-plane radiation pattern was lens than $-17 \mathrm{~dB}$, and the sidelobe level of the H-plane was less than $-12 \mathrm{~dB}$.

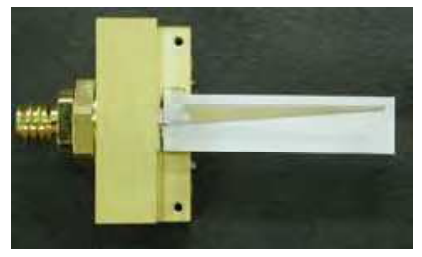

(a) Photo of antenna module

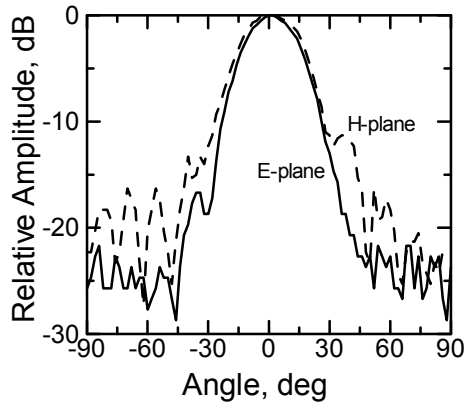

(b) Measured radiation pattern

Fig. 20. Antipodal LTSA

\subsection{Lens}

When designing a lens, designers have to consider spatial resolution, aberration, viewing field, and matching between the lens and the antenna.

The authors chose polyethylene as the lens material because it has high permeability for millimeter waves and is very workable. As the imager will be used in a short range between 1 and $20 \mathrm{~m}$, a lens with a diameter of $20 \mathrm{~cm}$ is sufficient for obtaining high spatial resolution. The authors used an even asphere function for the surface of the lens, and optimized 
parameters in that function to realize a small aberration on the focal plane. This optimization was done using ZEMAX.

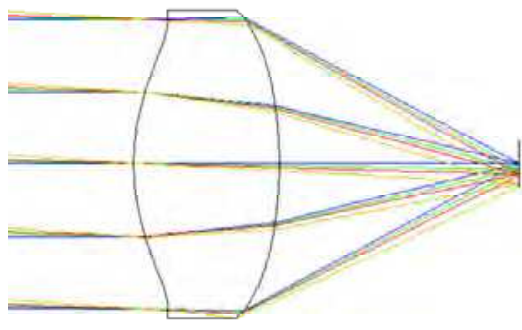

(a) Ray trace

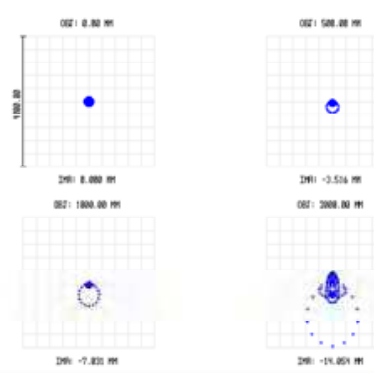

(b) Spot diagrams

Fig. 21. Ray trace and spot diagram of lens

\subsection{4-GHz-band millimeter-wave image}

Using the components described above, the authors developed a PMMW imaging test system. The metal pattern of the antipodal LTSA was printed on polyamide. And the receiver MMIC was mounted on an antenna substrate by a flip-chip bonding assembly. A DC amplifier and bias circuits are also mounted on the antenna substrate. A photo of the receiver module is shown in Fig. 22. The size was as small as $48 \times 12 \mathrm{~mm}^{2}$.

The temperature resolution and integration time of the receiver module is about $1 \mathrm{~K}$ and 10 msec. Moreover, the authors arrayed the receiver module by $10 \times 4$ to reduce the image collection time. The receiver array was set on the focal plane and scanned by a mechanical scanning system. The image acquisition time was $10 \mathrm{sec}$ for $40 \times 40$ pixels.

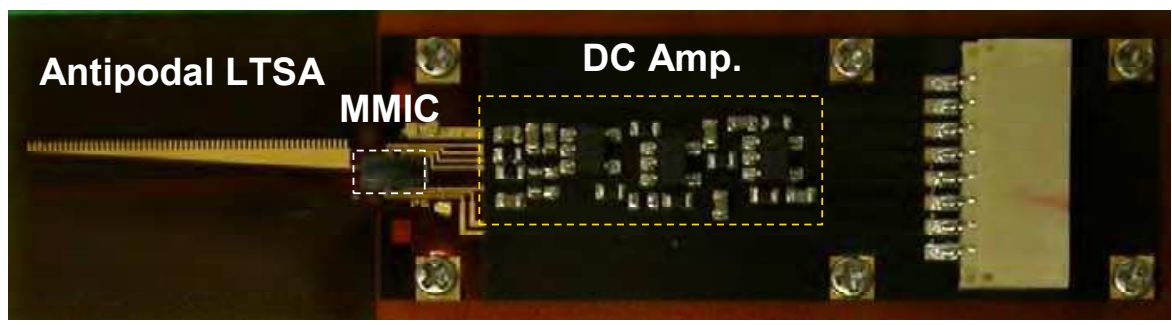

Fig. 22. Photo of the receiver module

The authors took images of a human indoors. The human was concealing a metallic object (simulating a gun). The distance between the human and the receiver was almost 2.5 meters. Fig. 23 shows the passive millimeter-wave image and corresponding photo and infrared image. The image size was $40 \times 40$ pixels, and the spot size on the target was $2 \mathrm{~cm}$. The blue area represents colder radiometric temperatures for the sample metal object and the ambient temperature, and the red areas represent warmer temperatures. As you can see, the 
millimeter-wave image is able to distinguish between the human and the concealed metal object.

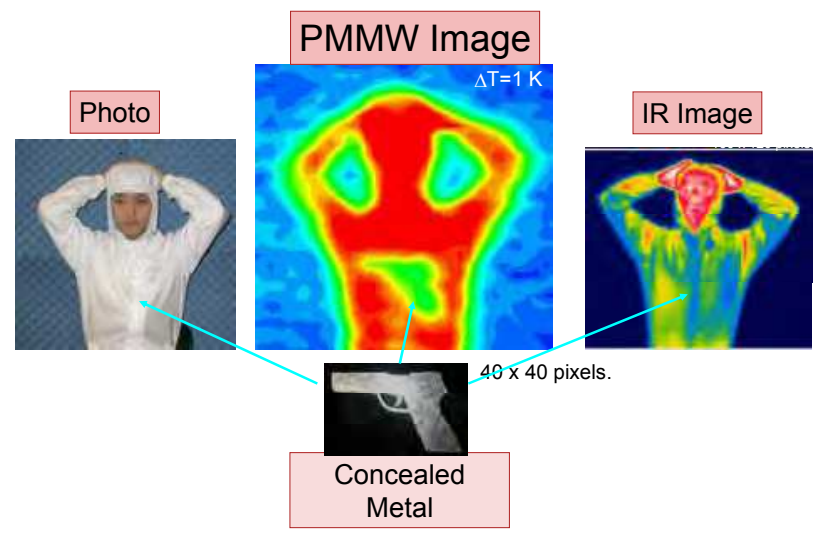

Fig. 23. Passive millimeter-wave image of a concealed metal object shown next to a photo and IR image

Part of this work was supported by the Ministry of Internal Affairs and Communications under the Strategic Information and Communications R\&D Promotion Programme (SCOPE).

\section{Conclusion}

In this chapter, the general principle and several systems for millimeter-wave sensors were discussed, and the state of development was also described. Furthermore, a 94-GHz-band passive millimeter-wave imaging sensor developed by Fujitsu Laboratories was shown in detail. The authors developed an ultra low-noise-receiver MMIC, and integrated it with a compact receiver module. The authors also realized a high-density imaging array to obtain millimeter-wave images in a short time. The considerations and the design method can also be used for other applications such as a broadband radio communication systems and radar.

\section{References}

Bhartia, P. \& Bahl, I., J. (1984). Millimeter Wave Engineering and Applications, John Wiley \& Sons, Itd., pp. 660-671

Kolinko, V. G.; Lin, S. H; Shek, A.; Manning, W.; Martin, C.; Hall, M.; Kirsten, O.; Moore, J. \& Wikner, D. A. (2005). A passive millimeter-wave imaging system for concealed weapons and explosives detection, Proc. SPIE, Vol. 5781, pp.85-92, USA

Kuki, T. (2008). RF world, No. 4 pp. 88-92, ASIN:B001GWJWN2, 2008

Millivision, (2009). http:/ / www.millivision.com/ portal-350.html

Mizuno, K.; Matano, H.; Wagatsuma, Y.; Warashina, H.; Sato, H.; Miyanaga, S. \& Yamanaka, Y. (2005). New applications of millimeter-wave incoherent imaging, Proc. IEEE MTT-S Int. Microwave Symp., pp. 629-632, June 2007, USA

Mizuno, K; Sato, H.; Hirose, T.; Sato, M.; Ohki, T. (2009). Development of Passive Millimeter-wave Imaging Sensors, $5^{\text {th }}$ SCOPE meeting, pp. 50-51, June 9, Tokyo 
Nakasha, Y.; Kawano, Y.; Suzuki, T.; Ohki, T.; Takahashi, T. Makiyama, K; Hirose, T. \& Hara, N. (2008). A W-band Wavelet Generator Using 0.13- $\mu \mathrm{m}$ InP HEMTs for Multi-gigabit Communications Based on Ultra-Wideband Impulse Radio, Proc. IEEE MTT-S Int. Microwave Symp. Digest, pp. 109-112, June 15-20, Atlanta, USA

Sato, H.; Sawaya, K.; Wagatsuma, Y.; \& Mizuno, K., (2004). Design of narrow width Fermi antenna with circular radiation pattern, Proc. IEEE Antennas and Progation Society Symp., pp. 4312-4315, June 2004, USA

Sato, M.; Hirose, T.; Ohki, T.; Sato H.; Sawaya K. \& Mizuno K. (2007). 94 GHz band highgain and low-noise amplifier using InP-HEMTs for passive millimeter wave imager, Proc. IEEE MTT-S Int. Microwave Symp., pp. 1775-1778, June 2007, USA

Sato, M.; Sato, H.; Hirose, T.; Ohki, T.; Takahashi, T.; Makiyama, K.; Kobayashi, H.; Sawaya, K. \& Mizuno, K., (2009). Compact receiver module for a $94 \mathrm{GHz}$ band passive millimetre-wave imager, IET Microw. Antennas Propag., Vol. 2, No. 9, pp. 848-853

Sato, M.; Hirose, T. \& Mizuno, K. (2009). Advanced MMIC Receiver for 94-GHz Band Passive Millimeter-wave Imager, IEICE TRANS. ELECTRON, Vol. E92-C, No. 9, pp. 1124-1129

Sinclair, G. N.; Anderton, R. N. \& Appleby, (2001). Outdoor passive millimetre wave security screening, 2001 IEEE 35 th International Carnahan Conference on Security Technology, pp. 172-179, Oct 16-19, London, UK

Tessmann, A.; Haydl, W. H.; Hulsmann, A. \& Schlechtweg, M. (1998). High-Gain Cascode MMIC's in Coplanar Technology at W-band Frequencies, IEEE Microwave and Guided wave Letters, Vol. 8, No. 12, Dec 1998, pp. 430-431

Tiuri, M. E. (1964). Radio astronomy receivers. IEEE Transactions on Antenna and propagation, Vol. 12, Issue 7, pp. 930-938

TSA. (2009). TSA Continues Millimeter Wave Passenger Imaging Technology Pilot, http:// www.tsa.gov/press/happenings/mwave_continues.shtm

Yujiri, L. (2006). Passive Millimeter Wave Imaging, 2006 IEEE MTT-S Int. Microwave Symp. Digest, pp. 98-101, June 13-16, USA

Vizard, D. R.; Doyle, R. (2006). Advances in Millimeter Wave Imaging and Radar Systems for Civil Applications, 2006 IEEE MTT-S Int. Microwave Symp. Digest, pp. 94-97, June 13-16, USA

Wills, M. (2009). Gaseous attenuation, http://www.mike-willis.com/Tutorial/gases.htm 
Microwave and Millimeter Wave Technologies: 


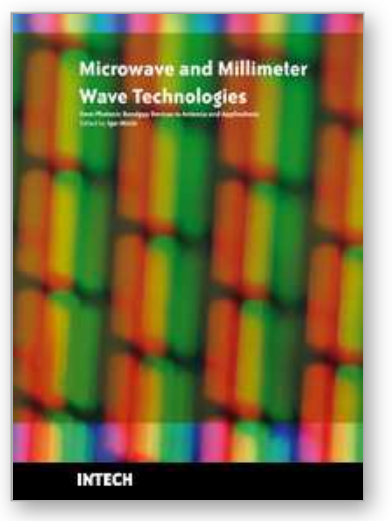

\section{Microwave and Millimeter Wave Technologies from Photonic Bandgap Devices to Antenna and Applications \\ Edited by Igor Minin}

ISBN 978-953-7619-66-4

Hard cover, 468 pages

Publisher InTech

Published online 01, March, 2010

Published in print edition March, 2010

The book deals with modern developments in microwave and millimeter wave technologies, presenting a wide selection of different topics within this interesting area. From a description of the evolution of technological processes for the design of passive functions in milimetre-wave frequency range, to different applications and different materials evaluation, the book offers an extensive view of the current trends in the field. Hopefully the book will attract more interest in microwave and millimeter wave technologies and simulate new ideas on this fascinating subject.

\section{How to reference}

In order to correctly reference this scholarly work, feel free to copy and paste the following:

Masaru Sato and Koji Mizuno (2010). Millimeter-Wave Imaging Sensor, Microwave and Millimeter Wave Technologies from Photonic Bandgap Devices to Antenna and Applications, Igor Minin (Ed.), ISBN: 978-9537619-66-4, InTech, Available from: http://www.intechopen.com/books/microwave-and-millimeter-wavetechnologies-from-photonic-bandgap-devices-to-antenna-and-applications/millimeter-wave-imaging-sensor

\section{INTECH}

open science | open minds

\section{InTech Europe}

University Campus STeP Ri

Slavka Krautzeka 83/A

51000 Rijeka, Croatia

Phone: +385 (51) 770447

Fax: +385 (51) 686166

www.intechopen.com

\section{InTech China}

Unit 405, Office Block, Hotel Equatorial Shanghai

No.65, Yan An Road (West), Shanghai, 200040, China

中国上海市延安西路65号上海国际贵都大饭店办公楼405单元

Phone: +86-21-62489820

Fax: $+86-21-62489821$ 
(C) 2010 The Author(s). Licensee IntechOpen. This chapter is distributed under the terms of the Creative Commons Attribution-NonCommercialShareAlike-3.0 License, which permits use, distribution and reproduction for non-commercial purposes, provided the original is properly cited and derivative works building on this content are distributed under the same license. 